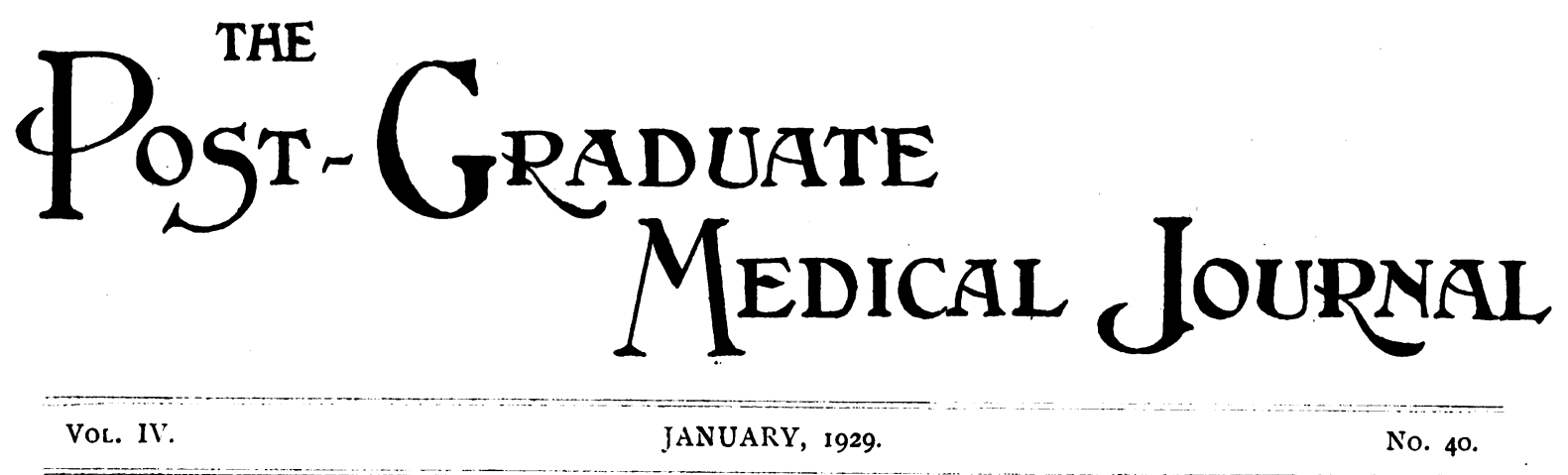

\title{
CONTENTS
}

PAGE

By H. W. Carson, F.R.C.S.

Somf Basic Remedies in the Treatment of Diseases of the Skin $\quad \ldots 60$

By Henry C. Semon, M.D., M.R.C.P.

$\begin{array}{llllllllll}\text { SOME Hamatological Notes } & \ldots & \ldots & \ldots & \ldots & \ldots & \ldots & \ldots & \ldots & 68\end{array}$

By Geoffrey Hadfilid.

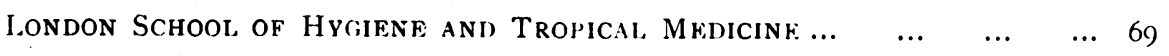

$\begin{array}{llllllllllll}\text { EDItORIAL NOtES } & \ldots & \ldots & \ldots & \ldots & \ldots & \ldots & \ldots & \ldots & \ldots & \ldots & 70\end{array}$

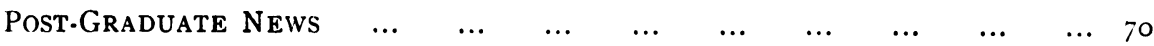

Fellowship of Medicine and Post-Graduate Meidcal Association.-

$\begin{array}{llllllllll}\text { SPECIAL COURSES } \ldots & \ldots & \ldots & \ldots & \ldots & \ldots & \ldots & \ldots & \ldots & \mathrm{v}\end{array}$

\section{INTESTINAL OBSTRUCTION}

\section{By H. W. CARSON, F.R.C:S.}

Senior Surgeon, Prince of Wales's Gencral Hospital, London.

Delizered to Rugby Medical Society, October 1, 1927.

I HAVE been asked to open a discussion on "Intestinal Obstruction," and in doing so I feel that the simplest way to approach so complicated a subject is to lay before you my own difficulties, in the hope that I may find that they are your difficulties also, and a free discussion may help in their solution. I think it will be agreed that of late years little progress has been made, as far at any rate as the saving of life is concerned, in the treatment of these cases, except in external hernia and intussusception, and we still find a mortality of anything from 40 per cent. to 50 per cent. among obstructions from any cause but these two. The improvement in the strangulated hernia statistics is due to earlier diagnosis and perhaps to the fact that strangulated hernia is now relatively uncommon, although it still accounts for more obstruction than all the other causes put together, while intussusception has a better prognosis because it is easily diagnosed, and surgical treatment is now universally adopted.

\section{SMall Intestinal OBstruction. Diagnosis.}

Although it may be more important to realize that an acute abdominal emergency is present, rather than to make an exact diagnosis, certain facts are at once apparent :- 
(I) The clinical features of small and large intestine obstruction are entirely different, though in the late stages differentiation may be difficult.

(2) The higher the obstruction the more acute the symptoms.

(3) The lower the obstruction, and therefore the less acute the symptoms, the more the signs.

In the ordinary case of acute small intestine obstruction the onset is characterized by severe pain, collapse and vomiting. The vomit is not characteristic at this stage, and the pain and shock are out of all proportion to the findings. The pain is generalized or perhaps centred on the umbilicus, so the patient cannot help you by indicating the site of the trouble, but the pain is a true colicky pain, coming and going with peristalsis. The abdomen is flaccid, for there is no peritonitis at this stage, not tender and not distended. The character of this onset helps to exclude many acute abdominal lesions, such as perforation of a riscus, acute peritonitis, appendicitis, and some others, but the very absence of signs makes it difficult at once to decide on operation. As time goes on the vomit changes its character, becoming at first bilious, and then brownish, but still not offensive, and the bowels are inoperative in spite of enemata. At this stage some distension appears, and it is important to note that while in high obstruction there may be no distension, the lower the obstruction is in the small intestines the sooner distension will appear. If you are fortunate it may be possible in obstruction of the lower ileum to find the early distended coil, generally in the hypogastrium, and this gradually extends until the typical ladder type of distension is seen. A word of caution may be introduced here, for in some old patients with a "tissue paper" abdominal wall, the coils of intestine, and even movements of the coil, may be apparent where there is no obstruction whatever.

In the next. stage, collapse has increased, vomiting is incessant and has become offensive, more or less generalized distension is present, and it is possible now to see that there is absolute constipation. It is at this stage that we see so many of these cases; and it is now almost too late, as a toxamia is present which cannot be remedied by any surgical operation. Of the source and nature of these toxins there have been many speculations which we may discuss with advantage. First let us get a clear mental picture of this last stage. The patient is conscious and understands all that is going on and that is said to him. He is apprehensive, and yet hardly seems to realize how ill he is. He is not particularly restless, but he has jerky movements, particularly purposeless movements of the hands, a condition which has been described as "pickin: at the bedclothes." $\mathrm{He}$ has a hluish-grey colour, his eyes are sunken and staring, and his forehead cold and moist. His lips are dry, and he constantly moistens them with his tongue. His tongue is dry and blackened. $\mathrm{He}$ is frequently bringing up mouthfuls of dark brown offensive material. Respirations are shallow, owing to abdominal distension, and the pulse is rapid and small. The blood-pressure falls steadily, and the temperature is subnormal. The extremities are cold and the hands cyanosed. The abdomen is generally distended and tympanitic. The abdominal wall is tense owing to the distension, but not rigid. There is no tenderness. Constipation is absolute. The patient has no pain. When death occurs, it is sudden and almost unexpected. It is evident that the patient has absorbed some poison which has been generated in the intestinal tract, and has been absorbed from it into the general circulation. But this poisoning is not always present in intestinal obstruction.

It may vary according to three considerations : (I) The type of obstruction; (2) personal idiosyncrasy; and (3) the length of time which has elapsed. With regard to these points the reaction of patients to 
obstruction varies very widely. We are all aware of those cases of what are called fulminating appendicitis. In these cases death follows a train of symptoms which is indistinguishable from the late stages of obstruction, the tragedy is complete perhaps in twenty-four or thirty-six hours, and the removal of the appendix with drainage does not seem to make the slightest difference. On the other hand we find cases of strangulated hernia in which the gravest injury is done to the gut, and yet in which symptoms of toxæmia are much delayed. A few months ago I operated on a very fat woman aged 58 , with a strangulated umbilical hernia of some days' standing, in which not only had the loop of the transverse colon, which was the part affected, beccme gangrenous, but the actual skin over the hernia was sloughing. She was not in the least clinically toxæmic, she stood resection of the gut well, and made an uninterrupted recovery. Then again we see cases of delayed ileus. Let me give you an example. Early in August this year, I was sent for into the country to see a medical man who had thirty-six hours before been seized with a most violent generalized abdominal pain. $\mathrm{He}$ tried various remedies, but after the second sleepless night of pain he sent for me. $\mathrm{J}$ found an advanced diffuse peritonitis secondary to a completely gangrenous appendix. I removed the appendix, drained the abdomen, and adopted the ordinary postoperative measures. He did well for two days, and then vomiting recurred with gastric dilatation, symptoms of toxæmia supervened, and in spite of high jejunostomy and other methods, he died. With regard to the time factor in the appearance of toxæmic symptoms, we must agree I think that toxæmia is a late symptom, and that the early symptoms of vomiting and shock are not toxæmic, at least they occur without the other concomitant symptoms, namely, sweating, dehydration, lowered blood-pressure, purposeless movements, and so on. It may be that the actual intestinal injury is enough to account for these early symptoms.

My reference to ileus following peritonitis and intestinal obstruction, all in the same breath as it were, will make you think that I draw no distinction between them, and that is true, for I believe that deaths in acute obstruction, in acute peritonitis, and perhaps in acute pancreatitis, are all due to the same or a similar cause. I am convinced that we must divide our cases of intestinal obstruction into those with or without toxæmic symptoms, and that if we remedy the local disorder in those without toxæmia, we will get a success, while if we are content merely to remedy the disorder in the toxæmic cases we shall get a failure. So we come down to this question: "What is this factor occurring in the later stages of intestinal obstruction, whether it be mechanical or paralytic, which causes death ?" The subject has been debated for nearly roo years, since Amusset in 1839 first suggested that death was due to absorption of the decomposing contents of the strangulated loop, and the discussion still goes on. There are two main contentions: (I) That toxins are formed owing to the perverted action of the intestinal mucosa, probably in the duodenum; and (2) that the toxins are formed by anaerobic bacteria in the strangulated loop. The first has been evolved mainly by American investigators, of whom Whipple is the leader, and the details are familiar to all of you. The second was brought before the profession in 1926 by Mr. B. W. Williams, from the Surgical Unit of St. Thomas's Hospital (British Journal of Surgery, vol. xiv, 1.926, p. 295). He noted the similarity between the clinical picture of patients dying of intestinal obstruction, and those dying of gas gangrene. His investigations proved that in the vomit, and in the obstructed loop, B. welchii and its toxins were present, and that the conditions could be experimentally produced in mice by the intramuscular injection of fluid from the obstructed loop which had been rendered 
bacteria free, and that the condition could be prevented by a protective injection of $B$. welchii antitoxin. He explains the fact that toxæmia does not occur in pyloric obstruction, and in large intestine obstruction by the Medical Research Commitlee's report that in acid media the $B$. welchii toxin is destroyed, and the bacillus would be rapidly outgrown by other organisms. Working on this hypothesis he administered anti-gas gangrene serum at St. Thomas's Hospital to all cases of diffuse peritunitis, or intestinal obstruction (excluding idiopathic intussusception in infants, and large intestine obstruction unless acute symptoms had intervened) as an addition to the ordinary operative treatment. The results were most encouraging, the mortality in 256 cases of acute appendicitis was $I \cdot I 7$ per cent., as opposed to the normal rate of 6.3 per cent., and in fifty-four cases of acute obstruction the mortality was 9.3 per cent., as opposed to a normal rate of $24^{\circ} 8$ per cent. He says that in peritonitis the clinical effect of the administration of the serum was striking and immediate. Restlessness was greatly diminished or abolished, the patient slept, cyanosis disappeared, the pulse-rate fell, the bowels acted and distension gradually disappeared.

The most recent hypothesis advanced is that of Mr. St. Leger Brockman, of Sheffield, in the Arris and Gale Lecture at the Royal College of Surgeons in March, 1927 (Lancet, I927, vol. ix, p. 3i7). He came to the conclusion from experiments done by Eisberg on the ligating of the intestine above and below the bile ducts, that it was the absence of the bile which causes the symptoms of toxæmia if the gut is obstructed, and that this explains the absence of toxæmia in pyloric obstruction. He treated thirteen extreme cases by the rectal administration of human bile in saline solution. He says that in most cases after the first rectal injection the vomiting stops forthwith, or is much diminished, restlessness disappears, the tongue becomes moist and clean, the abdomen becomes soft with the disappearance of the distension, and the general condition of the patient is altered entirely for the better. Here are three views as to the causation of toxæmia in mechanical or paralytic ileus, each requiring different treatment : the altered duodenal secretion theory necessitates a high jejunostomy, the gas gangrene theory necessitates the administration of an antitoxin, the deprivation of bile theory necessitates the administration of bile, and in each case in addition to the ordinary accepted surgical methods. I may say that I am not convinced by the deprivation of bile theory, which to my mind fails to explain many things, but I shall not neglect to test it thoroughly.

Now let me offer you some points in my own purely operative technique.

(I) The gut is obstructed by a remedial cause such as a band. I deal with the band, and I leave the intestinal contents to find their own way. If distension is extreme, I puncture the coils with a trocar and cannula, and close the puncture after evacuating the contained gas, and fluid. I am well aware that this only empties a small section, but it helps in closing the abdomen.

(2) The gut is irretrievably damaged. I resect the damaged portion, and do an end-to-end anastomosis.

(3) The obstructing factor cannot be dealt with, e.g., in late caseating tubercilous gland obstruction. I short circuit.

To sum up this point I am not in favour of small intestine fistula. However, I must tell you that there is a growing tendency in favour of two or many stage operations in small intestine obstruction, particularly in gangrenous strangulated hernia, and this method may yet be found to save more lives than immediate resection and anastomosis. With regard to post-peritonitic ileus, we must all of us have noticed that if during the course of a desperate struggle after an operation for gangrenous appendicitis, a 
fæcal fistula develops, the case generally does well. This fistula usually occurs in the cæcum, but sometimes in the last few inches of the ileum, and I have long practised from time to time a cæcostomy in desperate cases. I have not really been satisfied with my results, and this is obvious enough if the duodenal poison theory or the gas gangrene theory is correct. Neither have I sufficient success with high jejunostomy to make me a strong supporter of this treatment, and $I$ am still pinning my faith to the old method of intravenous administration of saline and glucose, with pituitary extract and turpentine enemata. If the close of glucose is high, I correct it by giving insulin. I am now using anti-gas gangrene serum as a routine, but it is too early to say whether I am satisfied with my results. I am also using gastric lavage quite early in cases of persistent vomiting, indeed I believe that in a number of cases it is possible to diagnose the occurrence of acute or subacute dilatation of the stomach before vomiting occurs. In the after-treatment of diffuse peritonitis this possibility must always be kept in mind, and it is to the epigastrium that the keenest attention must be paid during the routine abdominal examinations after operation.

And now a few words about large intestine obstruction.

\section{LARGE INTESTINAL OBSTRUCTION.}

The obstruction is nearly always due to a malignant stricture, and the left colon is affected. If you draw an imaginary line from the right anterior superior spine to the tip of the ninth rib on the left side, you will divide the abdomen roughly into two halves. The right half contains the cæcum, ascending colon, hepatic flexure, and the greater part of the transverse colon; the left half contains the splenic flexure, descending colon, iliac and pelvic colon. The colon in the right half is supplied with blood by the superior mesenteric artery, and therefore from a developmental standpoint can be linked with the small intestine. Growths affecting this part of the colon tend to be of the rapidly growing, florid, breakingdown type, which do not readily cause obstruction; while growths affecting the left half of the colon tend towaids the scirrhus type, small sclerosing ulcers which lead sooner or later to obstruction. The latter type of growth does not give rise to many symptoms except to gradually increasing constipation, and therefore they are not suspected in their early stage, and many of them are seen for the first time only when obstruction has occurred. It is important to remember that this type of obstruction differs much in its urgency from the small intestine obstruction which we have been considering, for while the patient is in pain and much distressed by intestinal distension, there is no vomiting, and no toxæmia such as we have seen in the earlier picture. Here again we can divide our patients into two categories: (I) Those who give a long history of frequently recurring distensions which they have been able to overcome by aperients; and (2) those who have no long history to give. The importance of this division is that in the longstanding cases the cæcum, al ways the weakest and most distensible part of the colon, as the result of constantly recurring back pressure, may have become thinned, inflamed, and even ulcerated, and may give way under the strain of complete obstruction, and in these cases no time should be wasted in attempts to relieve the obstruction by simple means, but operation should be done at once. In cases obstructed for the first time, or in those with only a short history, the correct treatment is to try, by stopping mouth feeding, by oil and soap enemata, and by morphia and atropine hypodermically, to relieve spasm, and to remove the material which is blocking the narrowed passage, for in some of these cases obstruction is due not actually to the growth, but to fæcal masses aided perhaps by odema of the 
mucosa closing the passage. This attempt must not be persisted in too long, for these patients do not bear starvation well, and the cæcum may be in a worse condition than one thinks. If operation is decided upon what shall we do?

Everyone is agreed now that primary resection of the growth with immediate restoration of the intestinal canal is bad surgery, so we need not consider that. But there has been a good deal of discussion as to whether one should explore the abdomen so as to know exactly where the growth is, and then to do what seems best, or whether one should content oneself with opening the abdomen in the right iliac fossa, and draining the cæcum. To this operation has been given the name of "blind cæcostomy." Those in favour of "blind caecostomy" argue that the patient is saved the shock of the handling of the intestines (there may be difficulty, if distension is marked, in returning the gut to the abdomen), and that, as the cæcum will probahly have to be drained in any case, it had better be done first as last. Those in favour of exploration main. tain that it is not always easy to differentiate small from large intestine obstruction in its later stages, that it is better to know exactly what one has to deal with, that if the growth is low down a sigmoidostomy is much more satisfactory, and does its work better than a cæcostomy, and that sometimes in left colon growth the jejunum, which normally lies in front of it, is implicated in the growth, and is the main cause of the obstruction. (I had a case of this sort three months ago.) My own feeling is against "blind cæcostomy," among other reasons, because I do not think a cæcostomy drains the colon well if the growth is in the sigmoid, and if the growth is inoperable a permanent cæcostomy leaves the patient in a miserable condition, and once made it is not easy to close and replace by a more convenient artificial anus.

As we are discussing obstruction, I will not go into the question of the treatment of the growth, but just ask the question, "When the enterostomy has been safely performed, how are we to decide when to proceed to the radical operation in operable growths?" While one must, of course, be influenced to some extent by the state of the growth, I am inclined to advise you not to hurry. At least three weeks will be required to allow the intestine to recover. Fortunately one does not often have to combat any grave degree of toxæmia, but the condition of the tongue will generally show that there has been some, and the tongue is the best guide in deciding on the patient's general condition. The appetite, too, often improves amazingly after the enterostomy, and the patient's condition will improve rapidly on the more generous diet he is willing to take.

\section{SOME BASIC REMEDIES IN THE IREATMENT OF DISEASES OF THE SKIN. By HENRY C. SEMON. M.D., M.R.C.P. \\ Lecture delivered for the Fellowship of Medicine November 12, 1928.}

Credulity is common to mankind, and it was a wise physician who advised his audience to "hurry to prescribe a new remedy while it is still effective." That epigram, as I read it, does not encourage an absolute conservatism, although the implication is cynical. No man can afford to sit still, and the doctor who steadily refuses to avail himself of weapons which the advance of allied sciences have placed in his hands runs the risk of superannuation before his time. In considering a subject for this lecture, I set myself the task of giving you something practical. It occurred to me that an approach to a dermatological thesis through the fair avenues of pharma- 